\title{
Improvement of energy substrate metabolism by late evening snack supplementation in patients with liver cirrhosis: a meta-analysis
}

This article was published in the following Dove Press journal:

Therapeutics and Clinical Risk Management

\author{
Jia Yao',* \\ Weijia $\operatorname{Han}^{2,3, *}$ \\ Xiaojing Ren' \\ Lili Yuan' \\ Jun $X u^{\prime}$ \\ Zhongping Duan ${ }^{2,3}$ \\ 'Department of Gastroenterology and \\ General Surgery, Shanxi Dayi Hospital, \\ Taiyuan, People's Republic of China; \\ ${ }^{2}$ Difficult \& Complicated Liver Diseases \\ and Artificial Liver Center, Beijing Youan \\ Hospital, Capital Medical University, \\ Beijing, People's Republic of China; \\ ${ }^{3}$ Beijing Municipal Key Laboratory of \\ Liver Failure and Artificial Liver \\ Treatment Research, Beijing, People's \\ Republic of China \\ *These authors contributed equally to \\ this work
}

\begin{abstract}
Aim: Malnutrition is one of the most common complications in patients with liver cirrhosis. Abnormal energy substrate metabolism may contribute to aggravation of malnutrition. Late evening snack (LESs) supplementation has been recommended as an intervention to reduce starvation time and improve nutritional status. Published studies have analyzed the effect of LESs on the branched-chain amino acid (BCAA)/tyrosine ratio (BTR) and oxidation rate of fat and carbohydrate in patients with liver cirrhosis.
\end{abstract}

Methods: We searched PubMed, Cochrane Library, Web of Science and Embase for relevant research from January 2000 to October 2018. The primary outcome for this analysis was changes in BTR and fat and carbohydrate oxidation in patients with liver cirrhosis.

Results: A total of 9 articles, containing 211 patients, were included in this analysis. The results supported that supplementation with BCAA-enriched LESs improved BTR, and longterm supplementation with BCAAs ( $>1$ month) may be more beneficial than short-term supplementation ( $<1$ month) in patients with liver cirrhosis. In addition, supplementation with BCAAs may increase the oxidation rate of carbohydrates and decrease the oxidation rate of fat. Furthermore, compared with liquid-enriched LESs, BCAA was a better choice for increasing the oxidation of carbohydrates and decreasing the rate of fat oxidation.

Conclusion: BCAA-enriched LES supplementation is an appropriate nutritional intervention to improve abnormal energy substrate metabolism, which may improve malnutrition in patients with liver cirrhosis. Further research is needed on the long-term benefit and improved survival in patients with liver cirrhosis.

Keywords: cirrhosis, late evening snack, branched-chain amino acid, energy metabolism

\section{Introduction}

Malnutrition is one of the most common complications in patients with liver cirrhosis and is associated with an increased risk of morbidity and mortality. ${ }^{1}$ As an important part of the comprehensive treatment of liver cirrhosis, nutritional intervention can help to improve the nutritional status and quality of life of patients with liver cirrhosis. ${ }^{2}$ The liver is an important organ for maintaining normal energy and nutrient metabolism. Therefore, it is important to explore appropriate nutritional interventions for patients with liver cirrhosis.

Abnormal energy substrate metabolism is characteristic of patients with liver cirrhosis, which may aggravate malnutrition. ${ }^{3}$ In particular, after overnight fasting, patients with liver cirrhosis show increased rates of fat oxidation and decreased rates of carbohydrate oxidation compared to normal controls. In addition, previous 
studies on the correlation between lifestyle and liver disease have shown that lower daily frequency of meals is associated with nonalcoholic fatty liver disease, which is a risk factor for the development of liver cirrhosis, and a high daily eating frequency is associated with healthy lifestyle. ${ }^{4,5}$ Therefore, increasing the frequency of eating is used as intervention. Late evening snacks (LESs), which add an extra meal before sleep, has been recommended as an intervention to reduce the starvation time and improve nutritional status. ${ }^{6,7}$

LESs are currently an effective method to improve the metabolic status of patients with end-stage liver disease. ${ }^{6}$ However, published studies have had small sample sizes. It is also unclear whether long-term intervention with LESs and whether branched-chain amino acids (BCAAs) are more suitable for patients with liver cirrhosis. In patients with liver cirrhosis, due to the decline in glycogen reserves and activated glutamine synthesis in muscle, the consumption of BCAAs increases, which can lead to an imbalanced ratio of BCAA to aromatic amino acids (AAAs). ${ }^{8}$ Clinically, the BCAA/tyrosine ratio (BTR) reflects the ratio of BCAAs to AAAs, and changes are closely related to liver dysfunction. ${ }^{9}$ Previous studies have shown that BTR is a prognostic factor for hepatocellular carcinoma. ${ }^{10}$ In addition, the rate of fat and carbohydrate oxidation is closely related to prognosis of cirrhosis. ${ }^{11}$ Therefore, this study summarizes the currently published literature that has analyzed the effects of LESs on BTR and the oxidation rate of fat and carbohydrate in patients with liver cirrhosis. It may provide evidence for the clinical application of LESs in patients with liver cirrhosis.

\section{Material and methods}

\section{Study selection}

This meta-analysis was conducted and reported according to the Preferred Reporting Items for Systematic Reviews and Meta-Analyses (PRISMA). ${ }^{12}$ We selected articles from January 2000 to October 2018 using the databases of PubMed, Cochrane Library, Web of Science and Embase. All of the articles were about LESs in patients with liver cirrhosis. The following search terms were used: ("late evening snack" [Title/Abstract] or "nocturnal nutritional supplementation" [Title/Abstract] or "nocturnal snack" [Title/Abstract] or "evening snack" [Title/Abstract] or "nocturnal meal" [Title/Abstract]) or ("bedtime snack" [Title/Abstract]) and ("Liver Cirrhosis" [Title/Abstract] or "Cirrhosis,Liver" [Title/Abstract]) or "Cirrhoses, Liver"[Title/Abstract] or "Liver Cirrhoses"
[Title/Abstract] or "Hepatic Cirrhosis" [Title/Abstract] or "Cirrhoses, Hepatic" [Title/Abstract] or "Cirrhosis, Hepatic" [Title/Abstract] or "Hepatic Cirrhoses"[Title/ Abstract]) or "Cirrhosis, Hepatic" [Title/Abstract] or "Hepatic Cirrhoses" [Title/Abstract] or "Fibrosis, Liver" [Title/Abstract] or "Fibroses, Liver" [Title/Abstract] or "Liver Fibroses" [Title/Abstract] or "Liver Fibrosis" [Title/Abstract]).

Two investigators (J.Y. and W.H.) conducted a preliminary search separately, deleted duplicate records, sifted through relevant headings and abstracts, and identified relevant terms for further evaluation. References to retrieved articles were also reviewed to identify other eligible studies.

The study protocol was approved by the Ethics Committee of Beijing YouAn Hospital, Beijing, China.

\section{Definition and study endpoints}

Liver cirrhosis was diagnosed by clinical and laboratory profiles and by histological examination of liver biopsy specimens. ${ }^{13}$ The primary endpoint of this study was whether BTR and oxidation rate of carbohydrate and fat were affected by LESs.

Data extraction and quality assessment

Two investigators (J.Y. and W.H.) extracted the following information from the selected researches independently: first author, year of publication, intervention of experimental group, total numbers of patients enrolled, time of intervention for each event, levels of BTR, and oxidation rate of carbohydrate and fat before and after intervention. When research on the same patients appeared in multiple articles, to avoid duplication of information, we selected the study with the largest sample.

The US Agency for Healthcare Research and Quality (AHRQ) was used to evaluate bias risks in each study.

\section{Study eligibility}

Inclusion criteria: liver cirrhosis was diagnosed on the basis of pathological examination findings and ChildPugh classification. Exclusion criteria: patients had a history of other organ diseases, such as chronic heart failure or chronic respiratory, pancreatic or renal diseases.

\section{Statistical analysis}

We used Review Manager 5.2 and Stata 12.0 software for statistical analysis. Differences were expressed as mean \pm standard deviation with $95 \%$ CI. Heterogeneity was tested using the $I^{2}$ statistic. Heterogeneity was considered to be low in 
studies with $I^{2}$ 25-50\%, moderate in studies with $I^{2} 50-75 \%$, and high in studies with $I^{2}>75 \% . I^{2}>50 \%$ represented significant heterogeneity. A fixed-effects model was used when study heterogeneity was not significant and a random-effects model when heterogeneity was significant. Begg's test was used to estimate publication bias and sensitivity analysis was used to test stability.

\section{Results}

\section{Study selection and characteristics}

The selection process is illustrated in Figure 1. A total of 9 articles met the inclusion criteria. ${ }^{14-22}$ The main characteristics of the included studies are described in Table 1. The metaanalysis included 221 patients from Japan, aged 42-85 years. One study was a randomized controlled trial (RCT) ${ }^{18}$ and the others were single-arm studies. Three of the 9 studies supplied a pack of LESs $(210 \mathrm{kcal}),{ }^{14,17,22}$ others gave a pack of LESs and 1 or more supplements during the daytime. Three of the 9 studies used long-term LESs ( $>1$ month $)^{14,15,17}$ and the others used short-term LESs. Six of the 9 studies reported changes in BTR $^{14-17,19,21}$ and 5 reported changes in fat and carbohydrate oxidation. $^{18-22}$

\section{Quality assessment}

Although one study was an RCT, the baseline characteristics of each group were not comparable. We, therefore, compared the changes before and after LES treatment in each group, and we divided this RCT into 3 groups of one-arm study. Assessment of the single-arm studies by the AHRQ methodology checklist is shown in Table 2.
All included single-arm studies described the source of data and patient inclusion and exclusion criteria clearly, provided detailed explanation for excluded data, and presented measurements for the primary study endpoints.

\section{Effects of less on BTR}

We selected 6 studies that measured the changes in BTR before and after LESs. ${ }^{14-17,19,21}$ BTR was increased after LESs (MD $=0.79,95 \% \mathrm{CI}[0.15,1.43])$. The heterogeneity was significant $\left(I^{2}=90 \%\right)$ with publication bias $(P<0.00001)$ (Figure 2A). In order to find the reason, subgroup analysis was performed by treatment period. Three of the studies had short-term LESs ( $<1$ month) compared with $>1$ month in the others. BTR in the longterm LES group was increased with no heterogeneity $\left(I^{2}=0 \%\right)$, but BTR in the short-term LES group was increased with high heterogeneity $\left(I^{2}=96 \%\right)$ (Figure $2 \mathrm{~B}$ ). The differences between the groups were significant $(P=0.02)$. The changes in BTR after long-term LESs were superior to that after short-term LESs.

In the long-term treatment subgroup, BACCs were given besides LESs in one study, ${ }^{15}$ and BACCs were given once a day as LESs in 2 studies. ${ }^{14,17}$ Subgroup analysis showed that BTR was increased in the two groups, but there was no difference between these 2 treatments $(P=0.48)$ (Figure 2C).

Analysis of sensitivity was conducted to evaluate the robustness of the effect. The results showed that sensitivity was low $(\mathrm{MD}=0.58,95 \% \mathrm{CI}[0.35,0.81]$, the range for all

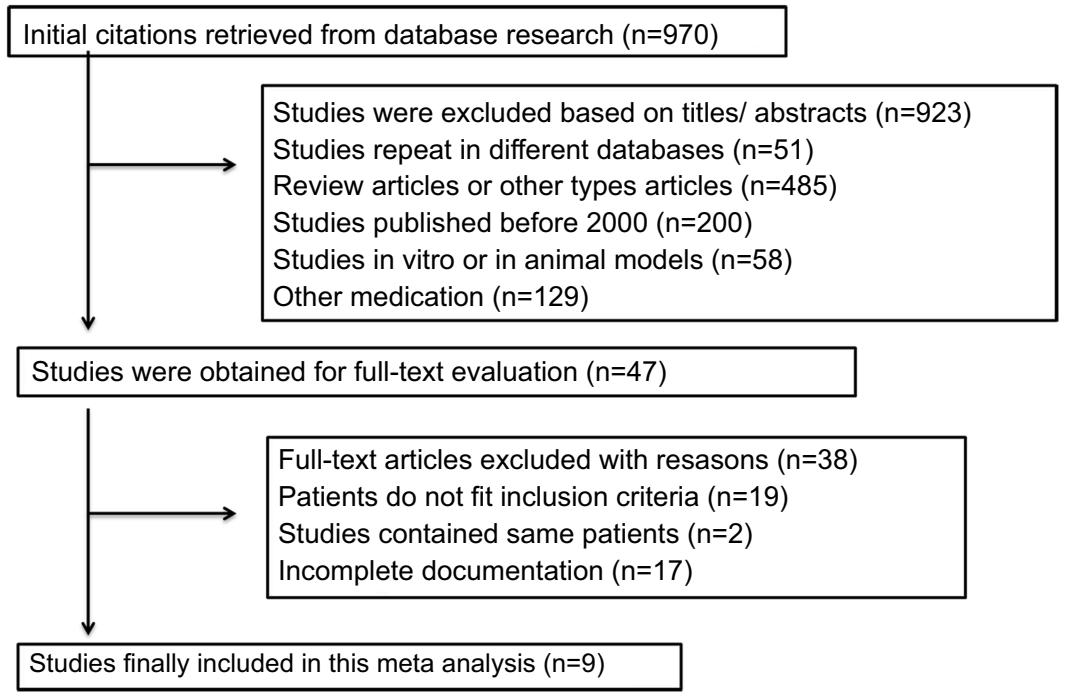

Figure I Flow diagram of study selection. 


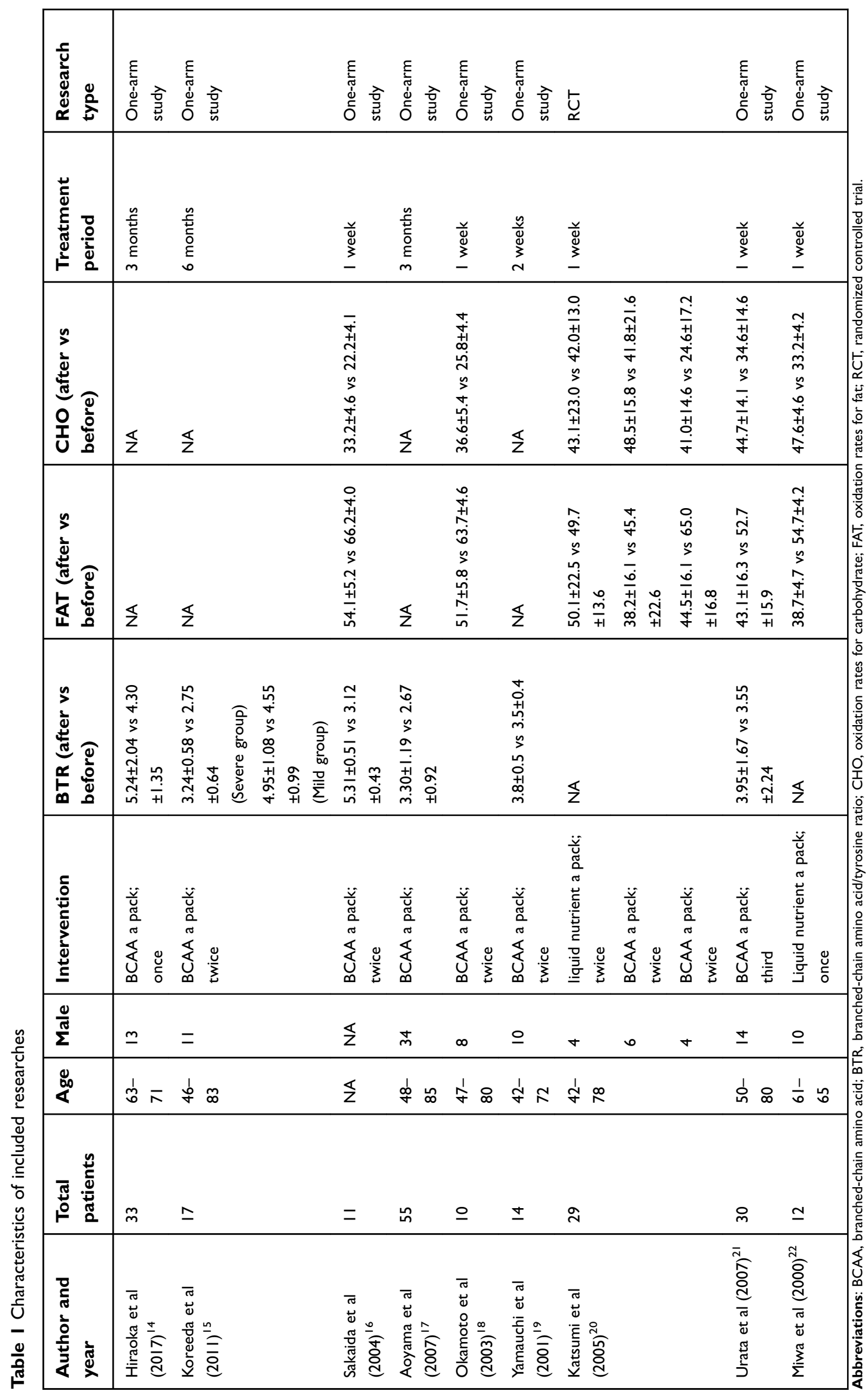




\begin{tabular}{|c|c|c|c|}
\hline 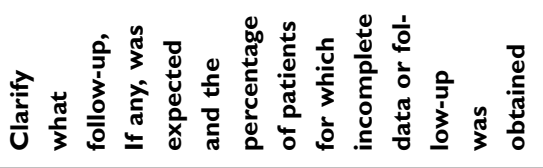 & $\stackrel{0}{z}$ z $\stackrel{0}{z} \quad \stackrel{0}{z}$ & $\stackrel{\circ}{z}$ & $\stackrel{\circ}{z} z_{z}^{\circ}$ \\
\hline 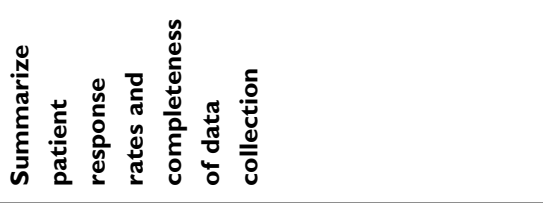 & 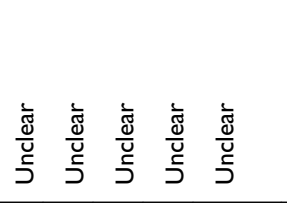 & 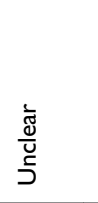 & 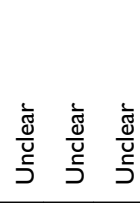 \\
\hline 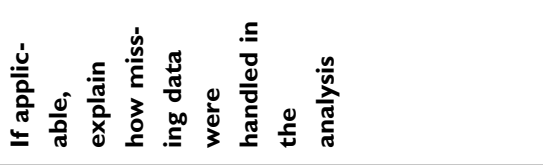 & 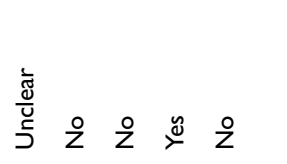 & o̊ & $\stackrel{0}{z} \stackrel{0}{z}$ \\
\hline 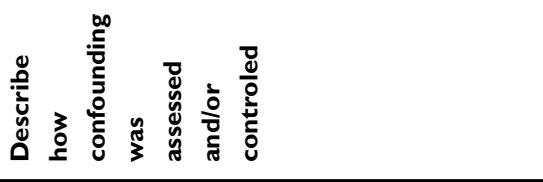 & 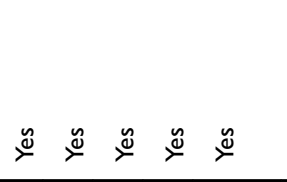 & 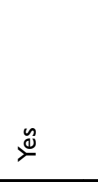 & $\stackrel{y}{\succ} \stackrel{y}{\longleftarrow} \stackrel{\tilde{\nu}}{\succ}$ \\
\hline 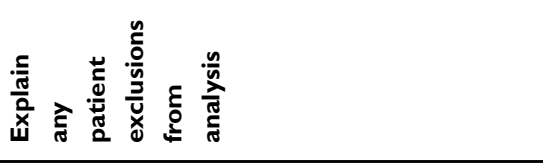 & $\stackrel{y}{z} \stackrel{0}{z} \quad \stackrel{0}{z} \quad \stackrel{0}{z}$ & $\stackrel{\circ}{z}$ & ż zำ \\
\hline 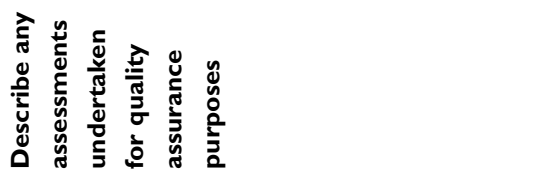 & 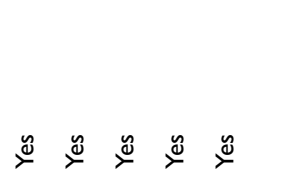 & $\stackrel{\check{\varpi}}{\check{\nu}}$ & ્ֻઁ \\
\hline 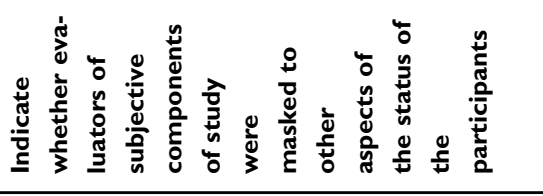 & 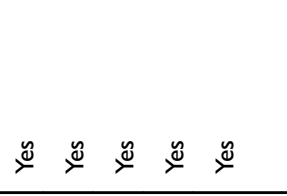 & $\stackrel{\varrho}{\check{\Perp}}$ & 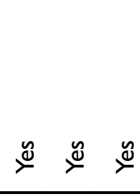 \\
\hline 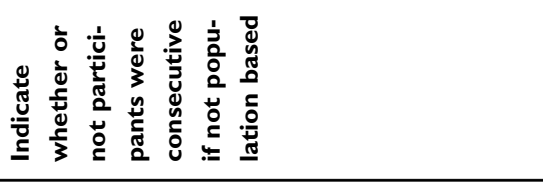 & 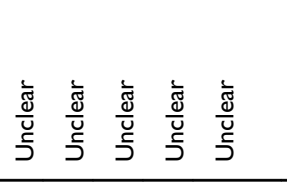 & 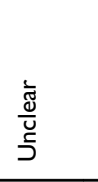 & 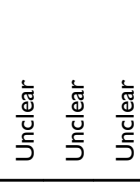 \\
\hline 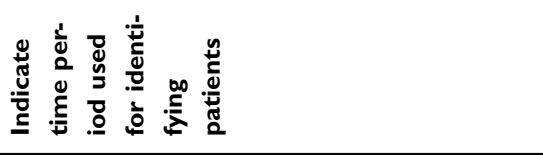 & 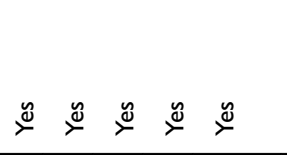 & $\stackrel{\Perp}{\check{\nu}}$ & $\stackrel{\Xi}{\nu} \stackrel{\Xi}{\longleftarrow}$ \\
\hline 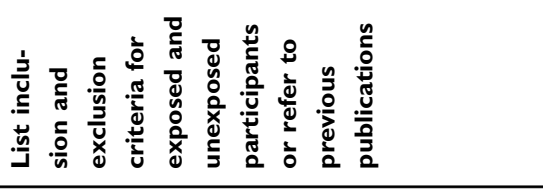 & 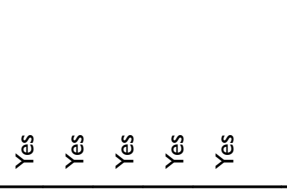 & $\stackrel{\Xi}{\simeq}$ & 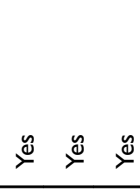 \\
\hline 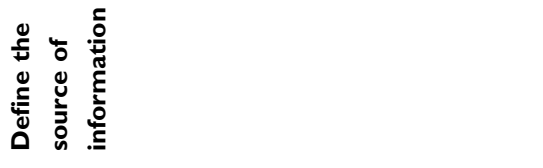 & 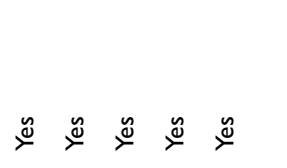 & $\stackrel{\mathscr{x}}{\tau}$ & 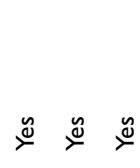 \\
\hline 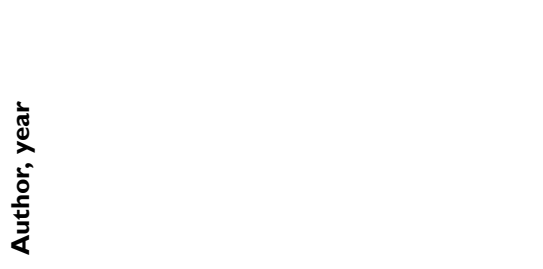 & 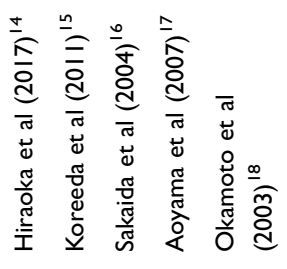 & 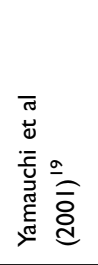 & 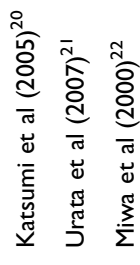 \\
\hline
\end{tabular}




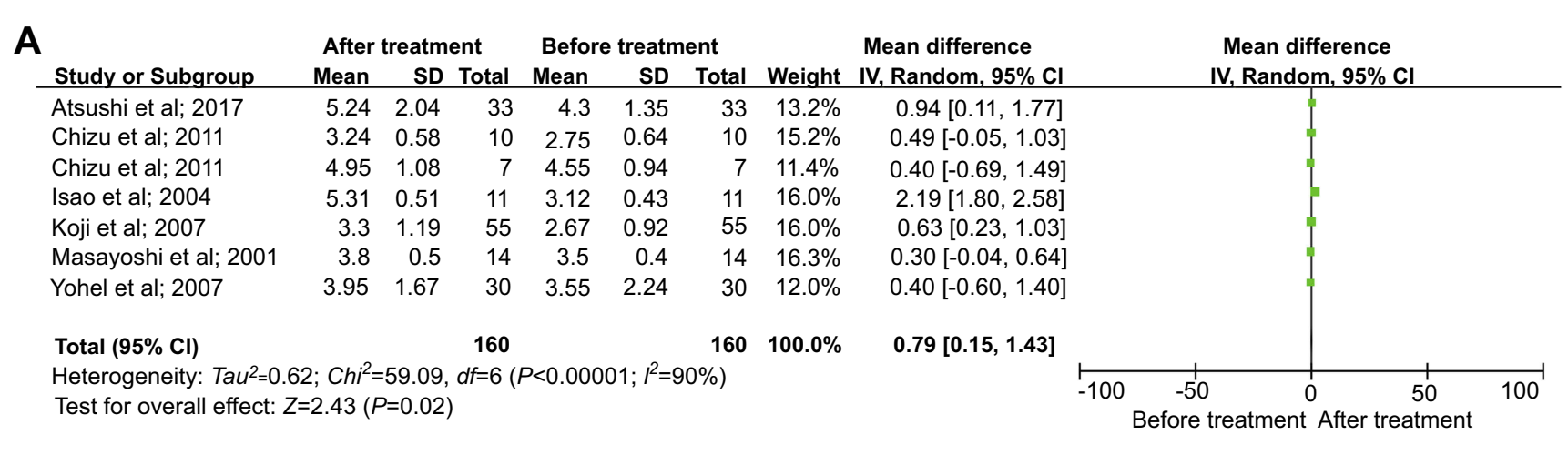

B

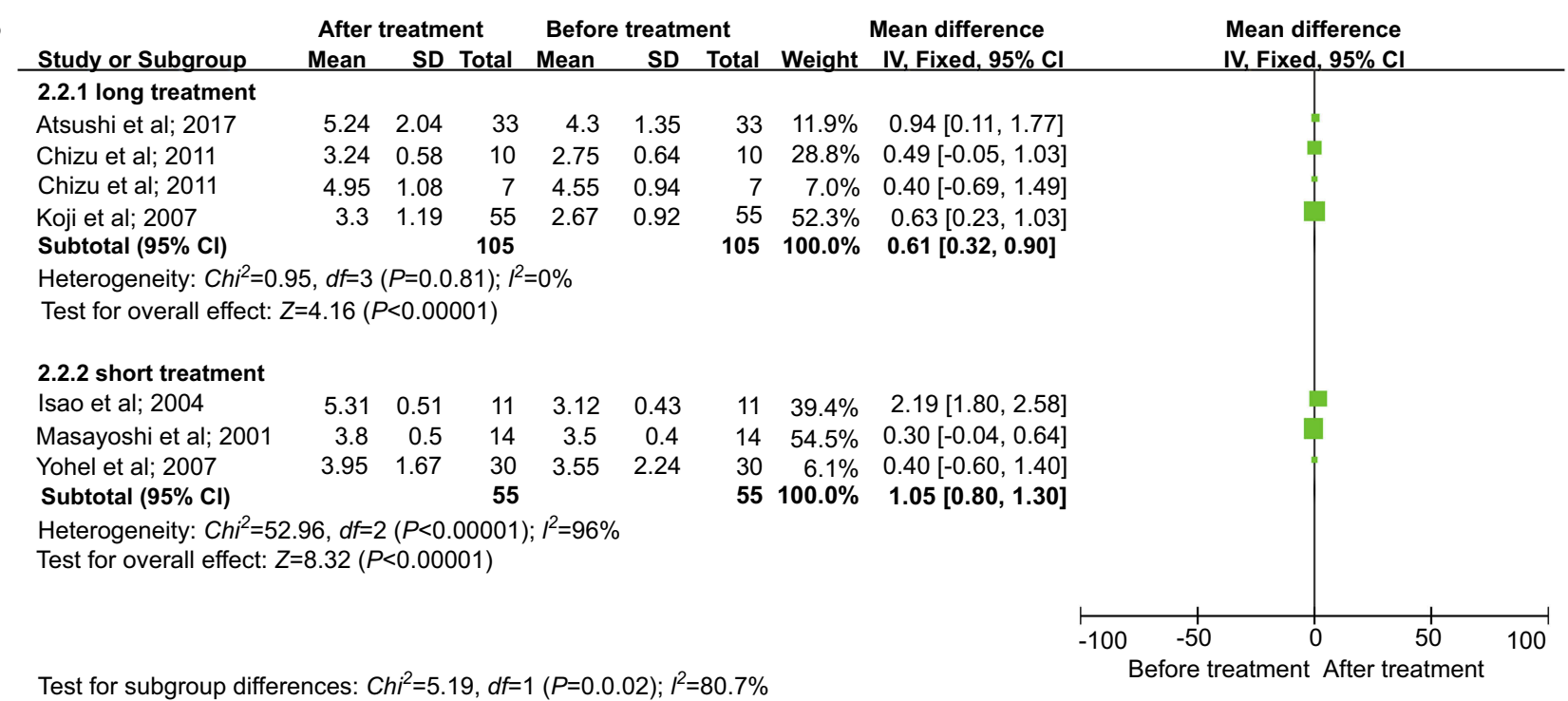

C

Test for subgroup differences: $C h i^{2}=5.19, d f=1(P=0.0 .02) ; l^{2}=80.7 \%$

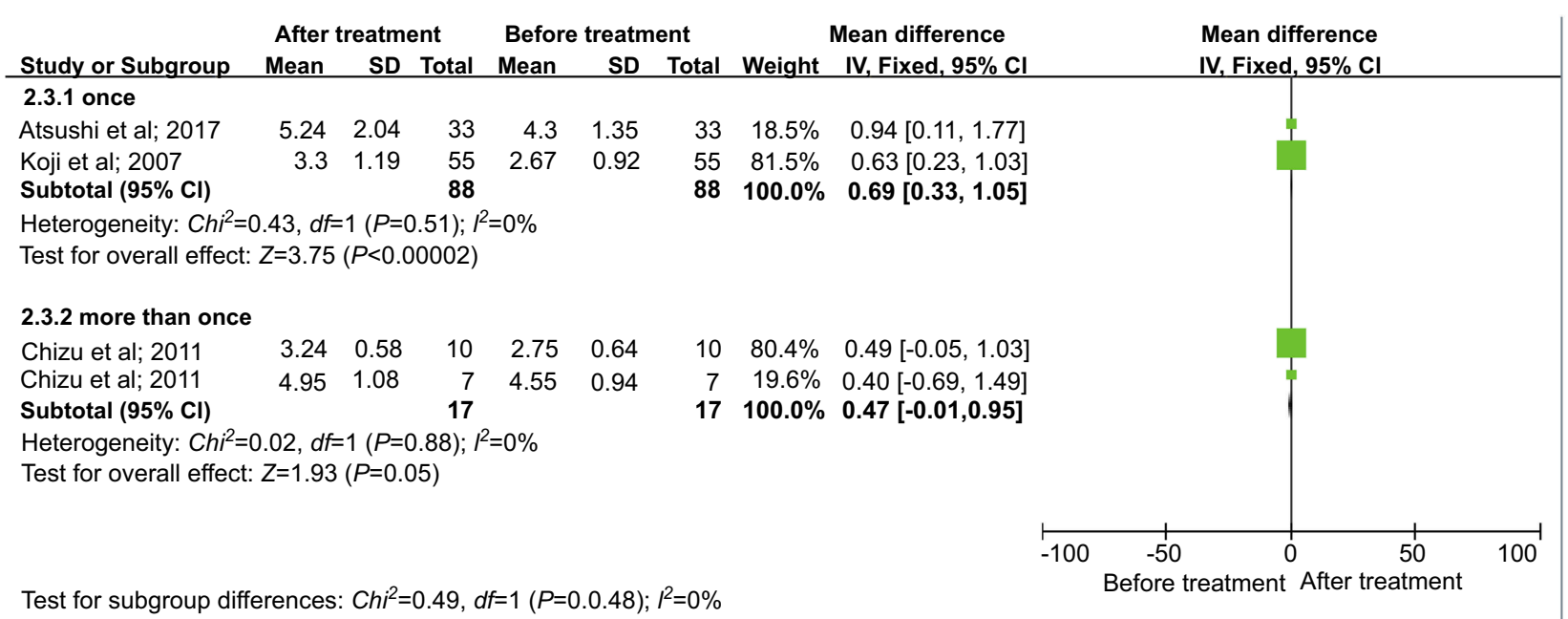

Figure 2 Meta-analysis of the changes in BTR. (A) Comparisons of BTR before and after LESs. (B) Subgroup analysis of the influence of different intervention periods on BTR. (C) Subgroup analysis of the effects of different daily intervention times on BTR.

Abbreviations: BTR, branched-chain amino acid/tyrosine ratio; LESs, late evening snacks. 
articles was 95\% CI [0.27, 0.93]). Begg's test showed publication bias in the 6 studies, although it was not significant $(\operatorname{Pr}>|z|=0.296$, continuity corrected).

\section{Effects of less on oxidation rate of fat}

We selected 5 studies that measured changes in fat oxidation before and after LESs. ${ }^{18-22}$ Fat oxidation was decreased after LESs $(\mathrm{MD}=-13.21,95 \% \mathrm{CI}$ $[-15.34,-11.07])$ with low heterogeneity $\left(I^{2}=23 \%\right)$ (Figure 3A).

Patients in two studies ${ }^{17,22}$ were treated with a pack of liquid nutrients as LESs, and the others received a pack of BCAAs. Subgroup analysis showed that BCAAs and liquid nutrients decreased fat oxidation, although there was no significant difference between the 2 groups $(P=0.15)$. There was no heterogeneity in the BCAA group, but the heterogeneity in the liquid nutrient group was significant $\left(I^{2}=73 \%\right.$ ) (Figure $\left.3 \mathrm{~B}\right)$, so this was an important source of heterogeneity. The results indicated BCAA was a better choice for reducing the oxidation rate of fat.

Analysis of sensitivity was conducted to evaluate the robustness of the effect. The results showed that sensitivity was low (mean estimate $=-1.11,95 \% \mathrm{CI}[-1.42,-0.80]$ ), and the range for all articles was $95 \% \mathrm{CI}[-1.78,-0.65])$. Begg's test showed publication bias in the 5 studies, although it was not significant $(\operatorname{Pr}>|z|=0.086$, continuity corrected).

\section{Effects of less on oxidation rate of carbohydrate}

We selected 5 studies that measured the oxidation rate of carbohydrate before and after LESs. ${ }^{18-22}$ The level of carbohydrate oxidation was increased after LESs $(\mathrm{MD}=11.92,95 \% \mathrm{CI}[9.88,13.96])$ with no heterogeneity $\left(I^{2}=0 \%\right)$ (Figure 4A).

Patients from two studies ${ }^{17,22}$ received LESs as liquid nutrients and the others received a pack of BCAAs.

\section{A}

\begin{tabular}{|c|c|c|c|c|}
\hline \multirow[b]{2}{*}{ Study or Subgroup } & \multicolumn{3}{|c|}{ After treatment } & \multirow{2}{*}{$\begin{array}{l}\text { Before } \\
\text { Mean }\end{array}$} \\
\hline & Mean & SD & Total & \\
\hline Isao et al; 2004 & 54.1 & 5.2 & 11 & 66.2 \\
\hline Mariko et al; 2003 & 51.7 & 5.8 & 10 & 63.7 \\
\hline Naoya et al; 2005 & 44.5 & 16.1 & 10 & 65 \\
\hline Naoya et al; 2005 & 38.2 & 16.1 & 9 & 45.4 \\
\hline Naoya et al; 2005 & 50.1 & 22.5 & 10 & 49.7 \\
\hline Yohei et al; 2007 & 43.1 & 16.3 & 30 & 52.7 \\
\hline Yoshiyuki et al; 2000 & 38.7 & 4.7 & 12 & 54.7 \\
\hline \multicolumn{3}{|l|}{ Total $(95 \% \mathrm{Cl})$} & \multicolumn{2}{|l|}{92} \\
\hline \multicolumn{5}{|c|}{$\begin{array}{l}\text { Heterogeneity: } C h i^{2}=7.77, d f=6(P=0.26) ; I^{2}=23 \% \\
\text { Test for overall effect: } Z=12.12(P<0.00001)\end{array}$} \\
\hline
\end{tabular}

$\begin{array}{rr}\text { SD } & \text { Tot } \\ 4 & \\ 4.6 & \\ 6.8 & \\ 2.6 & \\ 3.6 & \\ 5.9 & \\ 4.2\end{array}$

$\begin{array}{rr}11 & \text { Weight } \\ 10 & 30.3 \% \\ 10 & 21.7 \% \\ 9 & 1 . \\ 10 & 1.7 \% \\ 30 & 6.9 \% \\ 12 & 35.8 \% \\ & \\ 92 & \mathbf{1 0 0}\end{array}$

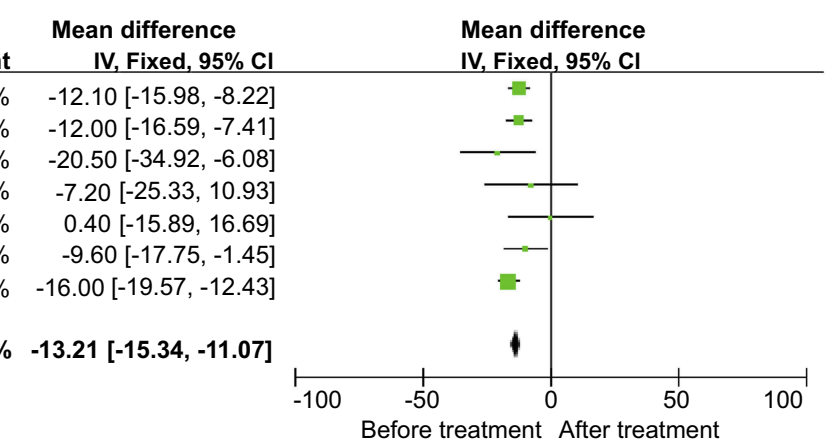

B

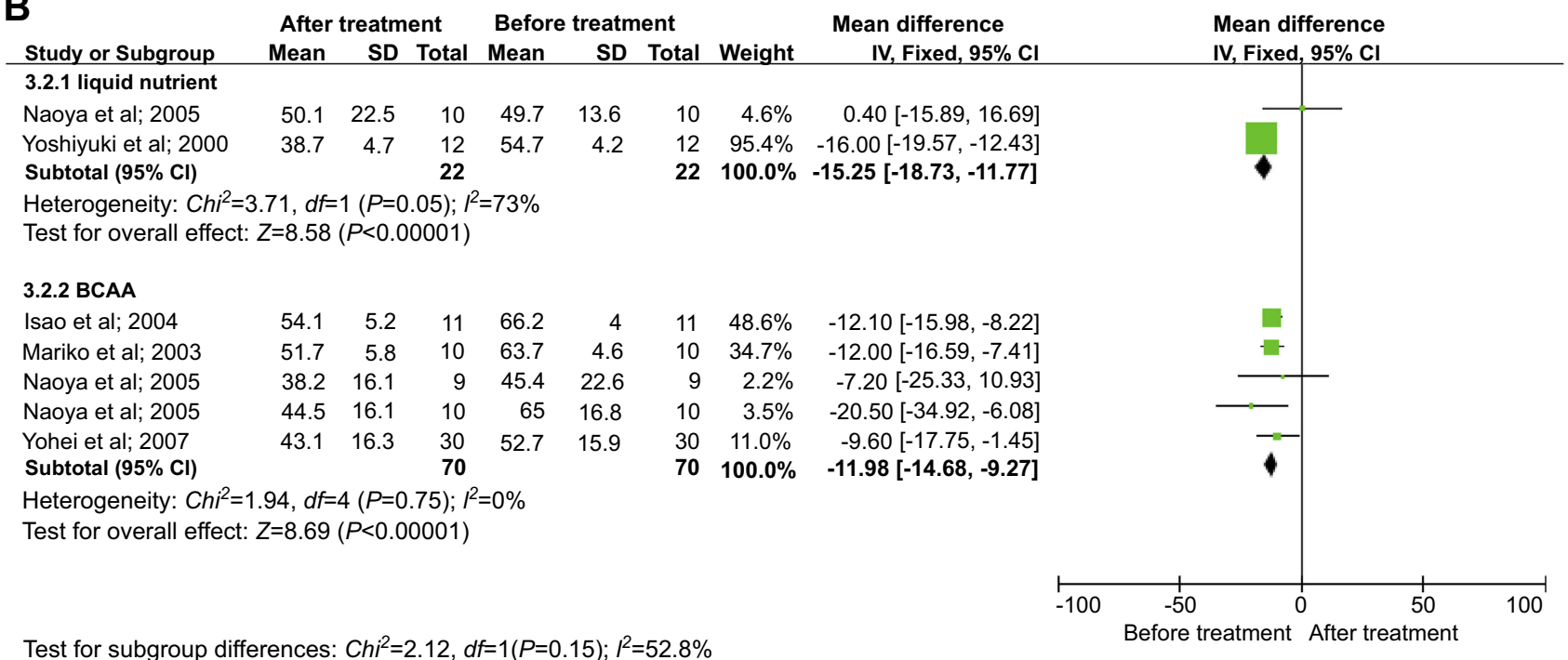

Figure 3 Meta-analysis of changes in the oxidation rate of fat. (A) Comparisons of the oxidation rate of fat before and after LESs. (B) Subgroup analysis of the effects of different interventions on fat oxidation.

Abbreviation: LESs, late evening snacks. 
A

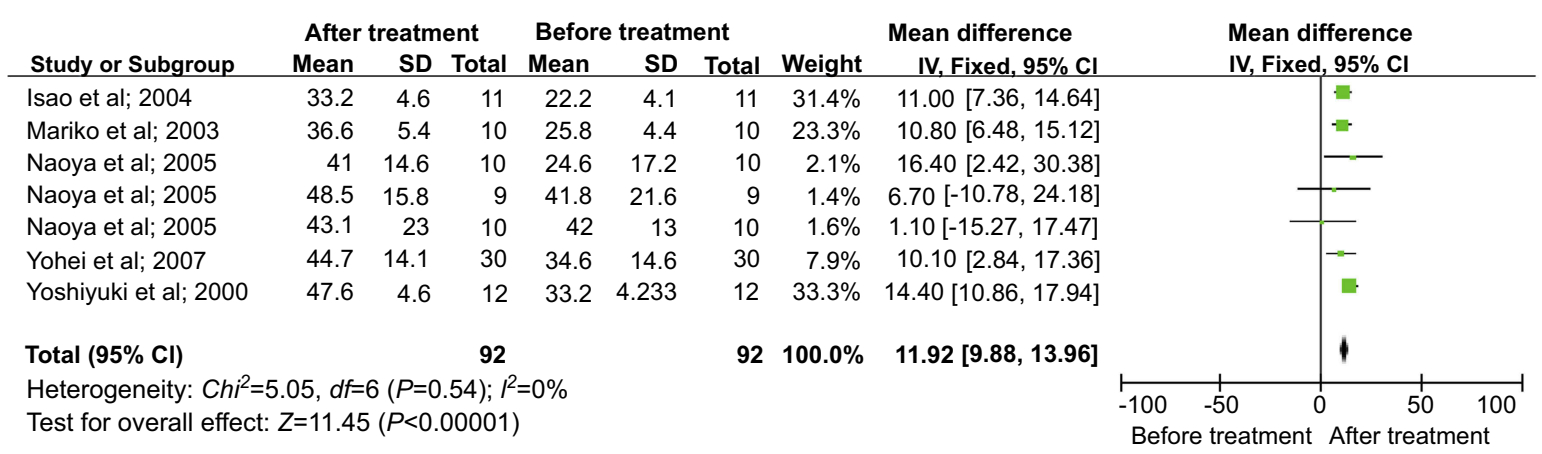

B

\begin{tabular}{llllrrrrr} 
B & \multicolumn{2}{c}{ After treatment } & \multicolumn{3}{c}{ Before treatment } & & Mean difference \\
\multicolumn{1}{c}{ Study or Subgroup } & Mean & SD & Total & Mean & SD & Total & Weight & IV, Fixed, 95\% Cl \\
\hline 4.2.1 liquid nutrition & & & & & & & & \\
Naoya et al; 2005 & 43.1 & 23 & 10 & 42 & 13 & 10 & $4.5 \%$ & $1.10[-15.27,17.47]$ \\
Yoshiyuki et al; 2000 & 47.6 & 4.6 & 12 & 33.2 & 4.233 & 12 & $95.5 \%$ & $14.40[10.86,17.94]$ \\
Subtotal (95\% Cl) & & & $\mathbf{2 2}$ & & & $\mathbf{2 2}$ & $\mathbf{1 0 0 . 0 \%}$ & $\mathbf{1 3 . 8 1}[\mathbf{1 0 . 3 5}, \mathbf{1 7 . 2 6}]$
\end{tabular}

Heterogeneity: $C h i^{2}=2.42, d f=1(P=0.12) ; l^{2}=59 \%$

Test for overall effect: $Z=7.83(P<0.00001)$

\subsubsection{BCAA}

Isao et al; 2004

Mariko et al; 2003

Naoya et al; 2005

Naoya et al; 2005

Yohei et al; 2007

Subtotal $(95 \% \mathrm{Cl})$

$\begin{array}{rr}33.2 & 4.6 \\ 36.6 & 5.4 \\ 48.5 & 15.8 \\ 41 & 14.6 \\ 44.7 & 14.1\end{array}$

Test for overall effect: $Z=8.46(P<0.00001)$

Test for subgroup differences: $C h i^{2}=1.76, d f=1(P=0.18) ; I^{2}=43.1 \%$

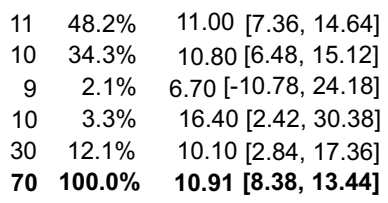

$10.91[8.38,13.44]$

Figure 4 Meta-analysis of changes in the oxidation rate of carbohydrate. (A) Comparisons of oxidation rate of carbohydrate before and after LESs. (B) Subgroup analysis of the effect of different interventions on the oxidation rate of carbohydrate.

Abbreviation: LESs, late evening snacks.

Subgroup analysis showed that BCAAs and liquid nutrients both increased the oxidation rate of carbohydrate, although there was no significant difference between the two treatment groups $(P=0.18)$. There was no heterogeneity in the BCAA group, but the heterogeneity in the liquid nutrient group was significant $\left(I^{2}=59 \%\right)$ (Figure 4B), so this was an important source of heterogeneity. The result indicated that BCAA was a better choice for increasing the oxidation rate of carbohydrate.

Analysis of sensitivity was conducted to evaluate the robustness of the effect. The results showed that sensitivity was low (MD $=1.09,95 \%$ CI $[0.78,1.4])$, the range for all articles was 95\% CI [0.63, 1.67]). Begg's test showed publication bias in the 5 studies, although it was not significant $(\operatorname{Pr}>|z|=0.086$, continuity corrected).

\section{Adverse effects of less}

There were no adverse events related to LESs in any of the 9 articles.

\section{Discussion}

This study evaluated the published literature on the effects of LESs, especially BCAA-based LESs. The results of the analysis supported that supplementation with BCAAs can improve BTR, and long-term supplementation with BCAAs (>1 month) may be more beneficial than shortterm supplementation $(<1$ month) in patients with liver cirrhosis. In addition, supplementation with BCAAs may increase the oxidation rate of carbohydrates and decrease the oxidation rate of fat, thereby significantly improving abnormal energy substrate metabolism in patients with liver cirrhosis. LESs are one of the commonly used nutritional interventions, but there is a scarcity of high-quality research among published studies. The results of the present study may provide evidence for BCAAs as a major component of LESs to improve substrate metabolism in patients with liver cirrhosis.

Since the glycogen reserve of liver cells in patients with cirrhosis is less than that of normal people, short- 
term hunger can induce gluconeogenesis. ${ }^{3}$ Therefore, after a natural sleep cycle, fat and protein are the main energysupplying substances, which is inclined to cause malnutrition in patients with liver cirrhosis. ${ }^{23,24}$ Therefore, to improve this condition, previous studies have proposed LESs as an intervention method, that is, giving a certain amount of calories at nighttime can reduce the time of hunger, thereby improving the abnormal substrate energy supply. ${ }^{25}$ Similar to previous studies, the present study supports the idea that LESs may improve the oxidation rate of carbohydrates and reduce the oxidation rate of fat in patients with liver cirrhosis. Furthermore, the BCAAbased LESs are better than fat-based LESs for increasing carbohydrate oxidation rate and reducing fat oxidation rate. Therefore, BCAA is considered to be a good choice for LES intervention.

Patients with liver cirrhosis often have amino acid metabolism disorder, which is often associated with a variety of complications such as hepatic encephalopathy and sarcopenia. ${ }^{14}$ Most studies have shown that BCAA supplementation improves amino acid profile, albumin level, and hepatic encephalopathy, ${ }^{26}$ and may also promote regeneration of liver cells. ${ }^{27}$ There is also research indicating that BCAAs can reduce the risk of liver cancer recurrence. ${ }^{28}$ However, to date, there has been no large study on BCAA supplementation in patients with liver cirrhosis. A total of 9 studies were pooled in the present study. The results showed that in patients with liver cirrhosis, BTR was significantly higher after than before oral BCAA supplementation. More importantly, there has been no study on the duration of BCAA supplementation. We showed that long-term BCAA supplementation $(>1$ month) may be more beneficial than short-term supplementation $(<1$ month) in patients with liver cirrhosis.

There were some limitations to this study, most of which were related to the quality of the research papers included. First, only 1 of the 9 studies was an RCT and the others were single-arm studies. Second, most of the studies had small samples. Third, most of the patients included in this study were Asians. Therefore, a large, multicenter RCT is needed to further analyze the effects of LESs, especially BCAAbased LESs, on patients with liver cirrhosis.

\section{Conclusion}

LESs based on BCAAs can improve BTR, increase the oxidation rate of carbohydrates, and decrease the oxidation rate of fat in patients with liver cirrhosis, which may improve the malnutrition in these patients. Long-term supplementation with BCAAs is more efficient and better than fat-based
LESs. Our results may provide evidence for the clinical application of LESs in patients with liver cirrhosis. When patients are supplemented with BCAAs, one should also consider the individual choice of the patient and the detailed status of their condition. Therefore, further research requires a larger sample of more individualized and standardized treatments.

\section{Acknowledgments}

This study was funded by the Shanxi Province 136 revitalization medical project (General Surgery Department); Science Foundation of China, Grant/Award Number: 81600561; National Key R\&D Program of China (No. 2017YFA0103000); Beijing Municipal Administration of Hospitals Clinical Medicine Development of Special Funding Support (No. ZYLX201806); “Beijing Municipal Administration of Hospitals" Ascent Plan (No. DFL201 51601); National Science and Technology Key Project on "Major Infectious Diseases such as HIV/AIDS, Viral Hepatitis Prevention and Treatment" (Nos. 2012ZX1000 2004-006, 2017ZX10203201-005, 2017ZX10201201-001001, 2017ZX10201201-002-002, 2017ZX10202203-006001, 2017ZX10302201-004-002).

\section{Disclosure}

The authors report no conflicts of interest in this work.

\section{References}

1. Tandon P, Raman M, Mourtzakis M, Merli M. A practical approach to nutritional screening and assessment in cirrhosis. Hepatology. 2017;65 (3):1044-1057. doi:10.1002/hep.29003

2. Mandato C, Di Nuzzi A, Vajro P. Nutrition and Liver Disease. Nutrients. 2017;10:1. doi:10.3390/nu10010009

3. Cheung K, Lee SS, Raman M. Prevalence and mechanisms of malnutrition in patients with advanced liver disease, and nutrition management strategies. Clinical Gastroenterol Hepatol. 2012;10(2):117-125. doi:10.1016/j.cgh.2011.08.016

4. Trovato FM, Catalano D, Musumeci G, et al. 4Ps medicine of the fatty liver: the research model of predictive, preventive, personalized and participatory medicine-recommendations for facing obesity, fatty liver and fibrosis epidemics. Epma J. 2014;5(1):21. doi:10.1186/1878-5085-5-20

5. Trovato FM, Martines GF, Brischetto D, et al. Fatty liver disease and lifestyle in youngsters: diet, food intake frequency, exercise, sleep shortage and fashion. Liver Int. 2016;36(3):427-433. doi:10.1111/ liv. 12957

6. Tsien CD, McCullough AJ, Dasarathy S. Late evening snack: exploiting a period of anabolic opportunity in cirrhosis. J Gastroenterol Hepatol. 2012;27(3):430-441. doi:10.1111/j.1440-1746.2011.06951.x

7. Plank LD, Gane EJ, Peng S, et al. Nocturnal nutritional supplementation improves total body protein status of patients with liver cirrhosis: a randomized 12-month trial. Hepatology. 2008;48(2):557-566. doi:10.1002/hep. 22367

8. Holecek M. Ammonia and amino acid profiles in liver cirrhosis: effects of variables leading to hepatic encephalopathy. Nutrition. 2015;31(1):14-20. doi:10.1016/j.nut.2014.03.016 
9. Furuichi Y, Imai Y, Miyata Y, et al. Branched-chain amino acidenriched nutrient increases blood platelet count in patients after endoscopic injection sclerotherapy. Hepatol Res. 2016;46(11):11291136. doi: $10.1111 /$ hepr. 12668

10. Ishikawa T. Branched-chain amino acids to tyrosine ratio value as a potential prognostic factor for hepatocellular carcinoma. World $J$ Gastroenterol. 2012;18(17):2005-2008. doi:10.3748/wjg.v18.i17.2005

11. Meng QH, Hou W, Yu HW, et al. Resting energy expenditure and substrate metabolism in patients with acute-on-chronic hepatitis B liver failure. J Clin Gastroenterol. 2011;45(5):456-461. doi:10.1097/ MCG.0b013e31820f7f02

12. Moher D, Shamseer L, Clarke M, et al. Preferred reporting items for systematic review and meta-analysis protocols (PRISMA-P) 2015 statement. Syst Rev. 2015;4:1. doi:10.1186/2046-4053-4-1

13. Tsochatzis EA, Bosch J, Burroughs AK. Liver cirrhosis. Lancet. 2014;383(9930):1749-1761. doi:10.1016/S0140-6736(14)60121-5

14. Hiraoka A, Michitaka K, Kiguchi D, et al. Efficacy of branched-chain amino acid supplementation and walking exercise for preventing sarcopenia in patients with liver cirrhosis. Eur J Gastroenterol Hepatol. 2017;29(12):1416-1423. doi:10.1097/MEG.0000000000000986

15. Koreeda C, Seki T, Okazaki K, et al. Effects of late evening snack including branched-chain amino acid on the function of hepatic parenchymal cells in patients with liver cirrhosis. Hepatol Res. 2011;41(5):417-422. doi:10.1111/j.1872-034X.2011.00795.x

16. Sakaida I, Tsuchiya M, Okamoto M, Okita K. Late evening snack and the change of blood glucose level in patients with liver cirrhosis. Hepatol Res. 2004;30S:67-72. doi:10.1016/j.hepres.2004.10.010

17. Aoyama K, Tsuchiya M, Mori K, et al. Effect of a late evening snack on outpatients with liver cirrhosis. Hepatol Res. 2007;37(8):608-614. doi:10.1111/j.1872-034X.2007.00036.x

18. Okamoto M, Sakaida I, Tsuchiya M, et al. Effect of a late evening snack on the blood glucose level and energy metabolism in patients with liver cirrhosis. Hepatol Res. 2003;27(1):45-50.

19. Yamauchi M, Takeda K, Sakamoto K, Ohata M, Toda. Effect of oral branched chain amino acid supplementation in the late evening on the nutritional state of patients with liver cirrhosis. Hepatol Res. 2001;21 (3):199-204.
20. Katsumi N, Kawamura N, Yamaguchi Y, et al. Effect of oral branched chain amino acid-rich nutrient administered during endoscopic injection sclerotherapy of cirrhotic patients. Hepatol Res. 2005;32(3):158-162. doi:10.1016/j.hepres.2005.03.013

21. Urata Y, Okita K, Korenaga K, et al. The Geffect of supplementation with branched-chain amino acids in patients with liver cirrhosis. Hepatol Res. 2007;37(7):510-516. doi:10.1111/j.1872034X.2007.00081.x

22. Miwa Y, Shiraki M, Kato M, et al. Improvement of fuel metabolism by nocturnal energy supplementation in patients with liver cirrhosis. Hepatol Res. 2000;18(3):184-189.

23. Changani KK, Jalan R, Cox IJ, et al. Evidence for altered hepatic gluconeogenesis in patients with cirrhosis using in vivo 31-phosphorus magnetic resonance spectroscopy. Gut. 2001;49(4): $557-564$.

24. Glass C, Hipskind $\mathrm{P}$, Tsien C, et al. Sarcopenia and a physiologically low respiratory quotient in patients with cirrhosis: a prospective controlled study. J Appl Physiol (1985). 2013;114(5):559-565. doi:10.1152/japplphysiol.01042.2012

25. Suzuki K, Endo R, Kohgo Y, et al. Guidelines on nutritional management in Japanese patients with liver cirrhosis from the perspective of preventing hepatocellular carcinoma. Hepatol Res. 2012;42(7):621-626. doi:10.1111/j.1872-034X.2012. 00990.x

26. Kawaguchi T, Taniguchi E, Sata M. Effects of oral branched-chain amino acids on hepatic encephalopathy and outcome in patients with liver cirrhosis. Nutr Clin Pract. 2013;28(5):580-588. doi:10.1177/ 0884533613496432

27. Beppu T, Nitta H, Hayashi H, et al. Effect of branched-chain amino acid supplementation on functional liver regeneration in patients undergoing portal vein embolization and sequential hepatectomy: a randomized controlled trial. J Gastroenterol. 2015;50 (12):1197-1205. doi:10.1007/s00535-015-1067-y

28. Nojiri S, Fujiwara K, Shinkai N, Iio E, Joh T. Effects of branchedchain amino acid supplementation after radiofrequency ablation for hepatocellular carcinoma: A randomized trial. Nutrition. 2017;33:2027. doi:10.1016/j.nut.2016.07.013
Therapeutics and Clinical Risk Management

\section{Publish your work in this journal}

Therapeutics and Clinical Risk Management is an international, peerreviewed journal of clinical therapeutics and risk management, focusing on concise rapid reporting of clinical studies in all therapeutic areas, outcomes, safety, and programs for the effective, safe, and sustained use of medicines. This journal is indexed on PubMed Central, CAS,
EMBase, Scopus and the Elsevier Bibliographic databases. The manuscript management system is completely online and includes a very quick and fair peer-review system, which is all easy to use. Visit http://www.dovepress.com/testimonials.php to read real quotes from published authors. 